\title{
Toxicologic evaluation of chronic feeding of glyphosate- resistant transgenic soybean GTS40-3-2 meal to rats
}

\author{
Jianqin Yuan', Zhongwei Tang', Jianghe Zhao', Zongyong Shi', Jundong Wang ${ }^{2 *}$ \\ ${ }^{1}$ College of Life Science, Shanxi Agricultural University, Taigu, Shanxi 030801, China, ${ }^{2}$ Shanxi Key Laboratory of Ecological Animal Science \\ and Environmental Veterinary Medicine, Shanxi Agricultural University, Taigu, Shanxi 030801, China
}

\section{A B S T R A C T}

\begin{abstract}
To assess the safety of glyphosate-resistant transgenic soybean, the transgenic soybean GTS40-3-2 meal and its non-transgenic counterpart parent A5403 were fed to first- and second-generation Sprague-Dawley (SD) rats (Rattus norvegicus) for 195 days. The soybean meal made up $20 \%$ of the total weight of the feed. Body weight, food consumption, hematology, blood biochemistry, and pathological observations were monitored weekly. The results showed that rats of each group experienced good growth and development, and although results of some organ weight ratios and serum biochemistry between the groups were significantly different $(P<0.05)$, they were of no biological significance because the variations were within the normal range. These results indicate that glyphosate-resistant transgenic soybean GTS40-3-2 meal is as safe as its non-transgenic parent soybean A5403 in a long-term SD rat feeding study. The study provides experimental evidence for the safe usage of transgenic soybean GTS40-3-2.
\end{abstract}

Keywords: Chronic feeding; Glyphosate-resistant transgenic soybean GTS40-3-2; Meal; Sprague-Dawley rat; Toxicology evaluation

\section{INTRODUCTION}

Because soybean is cheap and nutritious, it is not only one of the major food crops, but also a kind of important feed and industrial raw materials (He et al., 2009; Lu et al., 2014). However, the weeds result in a severe reduction in soybean quality and yield. Herbicides provide an effective way to prevent and control weeds, but they are most effective when used with herbicide-resistant soybean varieties. Although there are currently non-transgenic crops that are resistant against triazine, sulfonylurea, imidazolinone, and cyclohexanedione herbicides, most of the commercialized cultivars of the herbicide-resistant crops are genetically modified (Duke, 2005; Zhu, 2013). In 2013, transgenic crops were cultivated, reaching 175.2 million hectares worldwide (James, 2013). The herbicide-resistant mode of action is promoted by inserting five different genes. For example, the mutant 5-enolphyruvyl shikimate-3-phosphate synthase (EPSPS) gene and the glyphosate oxidoreductase (GOX) gene aid glyphosate resistance (such as CP4-EPSPS that is isolated from an Agrobacterium strain (P4), the bar gene aids glufosinate resistance, and the nitrilase gene aids bromoxynil resistance (Duke, 2005; Zhu, 2013).
Glyphosate is an active ingredient in many herbicides. By inhibiting the enzyme activity of the EPSPS, glyphosate suppresses the biosynthesis of aromatic amino acids. The enzyme of the EPSPS is encoded by the aro $A$ gene. With the mutant allele of aro $A$ located in Salmonella typhimurium, the EPSPS enzyme expression is not sensitive to glyphosate. Genetically modified (GM) full-fat soybean GTS40-3-2 is achieved by the introduction of this allele expression. The expressed enzyme confers tolerance to herbicide-glyphosate (Zhu, 2013).

In view of the rapid technological developments worldwide, China has taken action in research regarding transgenic plants (Zhu, 2013; Huang, 2015). Over the last decade, GM technique has become a popular topic; however, the public remains concerned about the safety of GM foods (Zhu, 2013). To investigate the safety of feeds or foods made from GM plants that are eaten by livestock and humans, a series of processes of safety assessment have been developed by international organizations (OECD, 1993, 1996, 1997; WHO, 1991, 1995; FAO/WHO, 2000; FAO, 1996; Zhu, 2013). In China, genetically modified crops are required to complete a $90-$

\footnotetext{
*Corresponding author:

Jundong Wang, Shanxi Key Laboratory of Ecological Animal Science and Environmental Veterinary Medicine, Shanxi Agricultural University, Taigu, Shanxi 030801, China. Tel.: +86 354 6288206, Fax: +86 3546222 942. E-mail: wangjd53@outlook.com
} 
day feeding study to determine safety. A large number of feeding studies on rodents use standard response variables and are designed to determine whether feeds containing GM crops are substantially equivalent to non-transgenic control feeds in nutrition and composition. Many GM crops have undergone studies that aim to detect adverse effects, including soybeans (Appenzeller et al., 2008; Chukwudebe et al., 2012; Sissener et al., 2009; Wang et al., 2013), tomatoes (Noteborn et al., 1995), cottonseed (Dryzga et al., 2007), rice (Kroghsbo et al., 2008), and maize grain (He et al., 2008, 2009; Zhu, 2013). The results showed that one or more GM genes were inserted into the plants' genomes without adverse effects.

The present study purpose was to investigate the unexpected potential impacts of soybean GTS40-3-2 on second-generation Sprague-Dawley (SD) rats. Rats were fed with containing 20\% GTS40-3-2 diets by weight. Rats of the control group were fed an untransformed parent A5403 soybean diets. The study examined gross, microscopic and pathology, and meatured parameters including feed intake, body weight, organ/body weight ratio, blood biochemistry, hematology, and toxicological response variables. The study was conducted in compliance with Chinese Standard GB15193.13-2003. In addition, the study was conducted at the Supervision, Inspection and Testing Center of the Ministry of Agriculture on Biological Product Ingredient (Taiyuan, China). The experimental protocols were approved by the Center for the Detection of Animal Ethics Committee.

\section{MATERIALS AND METHODS}

\section{Animals and housing}

Twelve five-week-old healthy SD rats were obtained from the Experimental Animal Center of Shanxi Medical University (Taiyuan, China) with weights of $90 \pm 10 \mathrm{~g}$. The rats were allowed a period of 1 week to adapt to their new environment prior to initiating the experiment. With a cycle of $12 \mathrm{~h}$ light/dark, they were housed at $22 \pm 2{ }^{\circ} \mathrm{C}$, and received a diet of non-GM soybean meal and ultrapure water ad libitum. The rats were randomly divided into four groups: male rats fed transgenic soybean GTS40-3-2 meal (GMM group, 2 rats), female rats fed transgenic soybean GTS40-3-2 meal (GMF group, 4 rats), male rats fed nonGM A5403 soybean meal (non-GMM group, 2 rats), and female non-GM rats fed non-GM A5403 soybean meal (non-GMF group, 4 rats). The four groups of animals were fed according to the national experimental animal feeding standard. After 6 weeks ( 3 months), the male and female rats were placed in the same cage (female/male ratio, 4:2). After being placed in the same cage, the time taken to breed the next generation was $\sim 21$ days. The second-generation rats were fed the corresponding GM and non-GM feeds after 3 weeks of nuring. After 2 weeks, 40 -week-old healthy SD rats, weighing 80-100 g, were obtained and randomly divided into four groups of 10: male and female rats fed transgenic soybean meal (GMM and GMF groups, respectively), and male and female rats fed nonGM soybean meal (non-GMM and non-GMF groups, respectively). Within 6 weeks, the GM and non-GM rats were weighed and data were recorded for a period of 90 days (total test time, 195 days).

\section{Plant materials}

The study used Monsanto's GM soybean GTS40-3-2 meal RR-resistance herbicide (glyphosate) and parent non-GM soybean meal A5403. The transgenic soybean GTS40-3-2 meal and non-GM A5403 soybean meal were verified by polymerase chain reaction. The Experimental Animal Center of Shanxi Medical University processed the standard rat feed (Chinese Standard GB14924.3-2010). The main nutrients of transgenic soybean GTS40-3-2 meal and the parent non-GM soybean A5403 meal are shown in Table 1 (Chinese Standard GB/T5009.6-2003, 5009.8-2008, 5009.3-5-2010). The feed formulations, which contain $20 \%$ soybean meal, are presented in Table 2.

\section{Clinical evaluation, feed consumption, and body weight measurement recording}

During the study, all rats were inspected daily. Clinical observations of noteworthy signs of toxicity, morbidity and mortality were recorded daily. Feed consumption and body weight were recorded weekly.

\section{Blood biochemistry}

Before blood samples collection, rats were fasted overnight for $16 \mathrm{~h}$ in order to minimize fluctuations in the parameters measured. Under anesthesia, blood samples of rats were obtained from the inner canthus without the administration of an anticoagulant. Using automatic Lx-20 analyzer of blood biochemistry (Beckman Coulter, Inc., Brea, CA, USA), the following plasma biochemical parameters were measured: blood urea nitrogen, creatine, glucose, albumin, total protein, cholesterol, triglycerides and enzyme activities of alkaline phosphatase, aspartate and alanine aminotransferase.

\section{Hematology}

Blood samples of rats were stabilized using heparin. Using XT-1800IV counter of blood cell (Sysmex Coporation, Kobe, Japan), hemoglobin, red blood cells, eosinophils, monocytes, platelet count, lymphocytes, and white blood cells were measured.

\section{Organ/body weight ratio}

Extraneous fat of rats in the brain, heart, lung, liver, kidneys, adrenal gland, spleen, testis, thymus, and ovaries 
was trimmed, and immediately weighed after removal. The study calculated the organ/body weight ratios.

\section{Gross pathology and histopathology}

All rats were visually inspected for gross pathology. Using standard histologic procedures, sections from all selected tissues of control and GTS40-3-2-fed rats were processed. For microscopic observation, representative samples of 14 tissues from the brain, heart, lung, liver, kidneys, adrenal gland, spleen, gastrointestinal tract (duodenum, jejunum, and ileum), testis, epididymis, uterus, and ovaries of rats were fixed in $4 \%$ buffered formaldehyde. Paraffinembedded tissues of approximately $5 \mu \mathrm{m}$ thickness were sectioned. With hematoxylin and eosin, they were stained.

\section{Statistical analysis}

Statistical software GraphPad Prism version 5.0 (Chinese Academy of Sciences statistics software, China) was applied to determine whether differences between the groups were attributable to the consumption of GTS403-2. Compositional data were presented as the mean \pm

\begin{tabular}{|c|c|c|}
\hline Nutrient & GTS40-3-2 meal & A5403 meal \\
\hline Moisture (\%) & $10.6 \pm 0.01$ & $11.1 \pm 0.01$ \\
\hline Crude protein (\%) & $45.1 \pm 0.07$ & $42.11 \pm 0.01$ \\
\hline Crude fiber (k/kg) & $5.9 \pm 0.10$ & $5.7 \pm 0.10$ \\
\hline Crude fat (k/kg) & $0.76 \pm 0.09$ & $3.0 \pm 0.11$ \\
\hline Ash $(\mathrm{k} / \mathrm{kg})$ & $5.80 \pm 0.07$ & $5.29 \pm 0.01$ \\
\hline $\mathrm{Ca}(\%)$ & $0.32 \pm 0.01$ & $0.26 \pm 0.01$ \\
\hline P (\%) & $0.65 \pm 0.01$ & $0.66 \pm 0.02$ \\
\hline
\end{tabular}

Table 2: Ingredients of feed

\begin{tabular}{lcc} 
Ingredients & $\begin{array}{c}\text { Ingredients of } \\
\text { transgenic soybean } \\
\text { GTS40-3-2 meal } \\
\text { feed (\%) }\end{array}$ & $\begin{array}{c}\text { Ingredients of } \\
\text { non-transgenic } \\
\text { soybean }\end{array}$ \\
A5403 meal feed (\%) \\
\hline $\begin{array}{l}\text { Transgenic } \\
\text { soybean pulp }\end{array}$ & 20.0 & - \\
Non-transgenic & - & 20.0 \\
soybean pulp & 25.0 & 25.0 \\
Maize & 20.0 & 20.0 \\
Bran & 21.4 & 21.4 \\
Wheat flour & 4.5 & 4.5 \\
Beet pulp & 1.7 & 1.7 \\
Plant oil & 1.6 & 1.6 \\
Calcium & & \\
hydrophosphate & 1.6 & 1.6 \\
Mountain flour & 1.5 & 1.5 \\
Fish meal & 1.0 & 1.0 \\
Yeast powder & 1.0 & 1.0 \\
Additive & 0.5 & 0.5 \\
Salt & 0.1 & 0.1 \\
Lysine & 0.1 & 0.1 \\
Methionine & & \\
\hline
\end{tabular}

confidence interval. Means were considered significantly different if their confidence intervals did not overlap. Data for male and female rats were analyzed separately, and results were presented as the mean \pm standard deviation, where appropriate. With one-way analysis of variance, statistical comparisons of the homogeneity of variance in body weight, clinical biochemistry, feed consumption, hematology, and organ weights between control and GTS40-3-2-fed rats were analyzed.

\section{RESULTS}

\section{Clinical observations}

All test rats survived during the feeding period. The clinical observations from all test rats were within normal ranges at all feeding times. No treatment-related abnormalities were observed in cage-side examinations of all rats (data not shown).

\section{Feed consumption and body weight}

Feed consumption and average weekly body weight gain were compared between male and female rats fed soybean GTS40-3-2 meal compared with rats fed non-transgenic soybean meal during the course of the feeding study. There were no any differences in feed consumption (Fig. 1) and average weekly body weight gain (Fig. 2) of rats.

\section{Organ/body weight ratios}

There were no treatment-related statistically significant differences or alterations detected in the organ/body weight ratios of female and male rats fed soybean GTS40-3-2 meal compared with rats fed non-transgenic soybean meal (Table 3). Males rats fed soybean GTS403-2 meal diets compared with rats fed non-transgenic A5403 soybean meal were found statistically significant decreases in the testis/body weight ratios $(P<0.05)$. Statistically significant decreases in ovary and brain/ body weight ratios were also observed in female rats fed soybean GTS40-3-2 meal diets compared with those fed non-transgenic soybean meal $(P<0.05)$. However, the weights were within their normal ranges, therefore these significant differences might be an accidental phenomenon.

\section{Blood biochemistry}

No group-related statistically identified alterations were found in the clinical blood biochemistry parameters for female and male rats fed transgenic soybean GTS40-3-2 meal compared with rats fed the non-transgenic soybean meal (Table 4).

\section{Hematology}

There were no group-related statistically identified changes or significant differences observed in the hematologic 
Table 3: Organ/body weight ratio (mean value \pm standard deviation, $n=10$ )

\begin{tabular}{lcccc} 
Organ & GMM group & Non-GMM group & GMF group & Non-GMF group \\
\hline Heart & $0.291 \pm 0.03$ & $0.297 \pm 0.02$ & $0.327 \pm 0.04$ & $0.330 \pm 0.03$ \\
Liver & $2.261 \pm 0.17$ & $2.234 \pm 0.11$ & $2.548 \pm 0.11$ & $2.723 \pm 0.14$ \\
Spleen & $0.139 \pm 0.01$ & $0.140 \pm 0.04$ & $0.166 \pm 0.01$ & $0.150 \pm 0.02$ \\
Lung & $0.300 \pm 0.02$ & $0.331 \pm 0.04$ & $0.400 \pm 0.09$ & $0.481 \pm 0.03$ \\
Adrenal & $0.085 \pm 0.01$ & $0.079 \pm 0.02$ & $0.108 \pm 0.02$ & $0.109 \pm 0.03$ \\
Kidneys & $0.615 \pm 0.03$ & $0.616 \pm 0.07$ & $0.690 \pm 0.01$ & $0.700 \pm 0.02$ \\
Thymus & $0.110 \pm 0.01$ & $0.120 \pm 0.02$ & $0.161 \pm 0.01$ & $0.173 \pm 0.03$ \\
Testis/Ovaries & $0.592 \pm 0.03^{\mathrm{a}}$ & $0.670 \pm 0.06$ & $0.049 \pm 0.02^{\mathrm{b}}$ & $0.060 \pm 0.01$ \\
Brain & $0.350 \pm 0.03$ & $0.430 \pm 0.04$ & $0.615 \pm 0.02^{\mathrm{b}}$ & $0.692 \pm 0.03$ \\
\hline
\end{tabular}

aSignificant difference between the GMM and non-GMM $(P<0.05)$, ${ }^{b}$ Significant difference between the GMF and non-GMF groups $(P<0.05)$, GMF, female rats fed transgenic soybean meal; non-GMF, female rats fed non-transgenic soybean meal; GMM group, male rats fed transgenic soybean meal; non-GMM, male rats fed non-transgenic soybean meal

Table 4: Blood biochemistry (mean value \pm standard deviation, $n=10$ )

\begin{tabular}{lcccc} 
Blood biochemistry & GMM group & Non-GMM group & GMF group & Non-GMF group \\
\hline ALT $(\mathrm{U} / \mathrm{L})$ & $47.00 \pm 4.00$ & $48.00 \pm 4.00$ & $44.00 \pm 5.00$ & $49.00 \pm 5.00$ \\
AST $(\mathrm{U} / \mathrm{L})$ & $279.00 \pm 13.00$ & $279.00 \pm 7.00$ & $288.00 \pm 20.00$ & $280.00 \pm 29.00$ \\
ALP $(\mathrm{U} / \mathrm{L})$ & $98.00 \pm 10.00$ & $99.00 \pm 15.00$ & $71.00 \pm 16.00$ & $82.00 \pm 18.00$ \\
BUN $(\mathrm{mmol} / \mathrm{L})$ & $9.60 \pm 0.80$ & $9.30 \pm 1.10$ & $8.70 \pm 0.60$ & $8.80 \pm 1.20$ \\
CRE $(\mu \mathrm{mol} / \mathrm{L})$ & $56.20 \pm 9.10$ & $55.10 \pm 6.80$ & $56.70 \pm 9.20$ & $56.90 \pm 8.10$ \\
GLU (mmol/L) & $5.01 \pm 0.73$ & $5.30 \pm 0.76$ & $5.75 \pm 0.36$ & $5.70 \pm 0.71$ \\
ALB (g/L) & $41.80 \pm 2.10$ & $43.10 \pm 1.60$ & $36.80 \pm 1.30$ & $36.30 \pm 1.30$ \\
TPR (g/L) & $72.50 \pm 2.30$ & $73.10 \pm 5.40$ & $70.50 \pm 2.70$ & $71.80 \pm 2.90$ \\
CHO (mmol/L) & $1.95 \pm 0.21$ & $1.88 \pm 0.17$ & $1.93 \pm 0.22$ & $1.94 \pm 0.32$ \\
TG (mmol/L) & $0.53 \pm 0.09$ & $0.51 \pm 0.10$ & $0.53 \pm 0.04$ & $0.54 \pm 0.09$ \\
\hline
\end{tabular}

ALT, alanine aminotransferase; BUN, blood urea nitrogen; AST, aspartate aminotransferase; CRE, creatine; ALP, alkaline phosphatase; GLU, glucose; ABL, albumin; TPR, total protein; CHO, cholesterol; TG, triglycerides; GMF, female rats fed transgenic soybean meal; non-GMF, female rats fed non-transgenic soybean meal; GMM, male rats fed transgenic soybean meal; non-GMM, male rats fed non-transgenic soybean meal

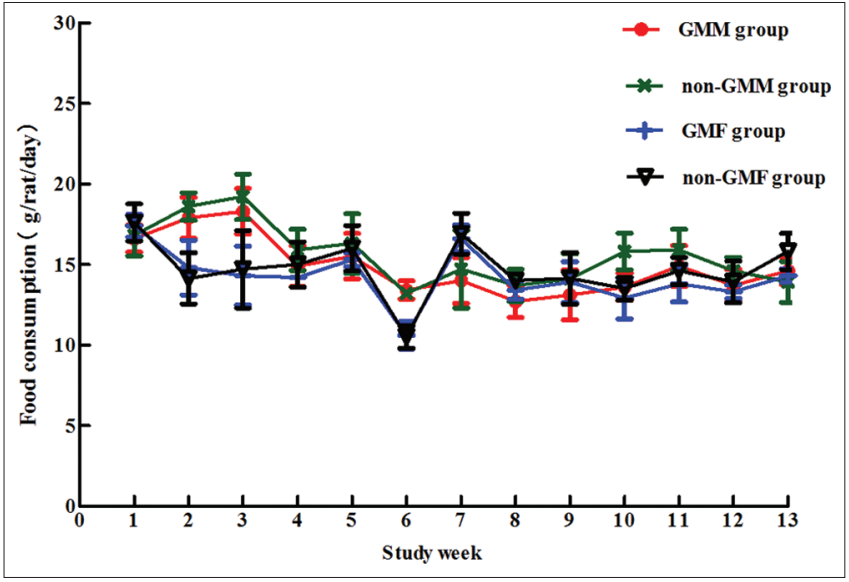

Fig 1. Average weekly feed consumption of rats. GMF, female rats fed transgenic soybean meal; non-GMF, female rats fed non-transgenic soybean meal; GMM, male rats fed transgenic soybean meal; nonGMM, male rats fed non-transgenic soybean meal.

parameters for female and male rats fed transgenic soybean GTS40-3-2 meal compared with rats fed non-transgenic soybean meal (Table 5).

\section{Clinical pathology}

No treatment-related histological or macroscopic effects were found in any rats in this study. No any pathological

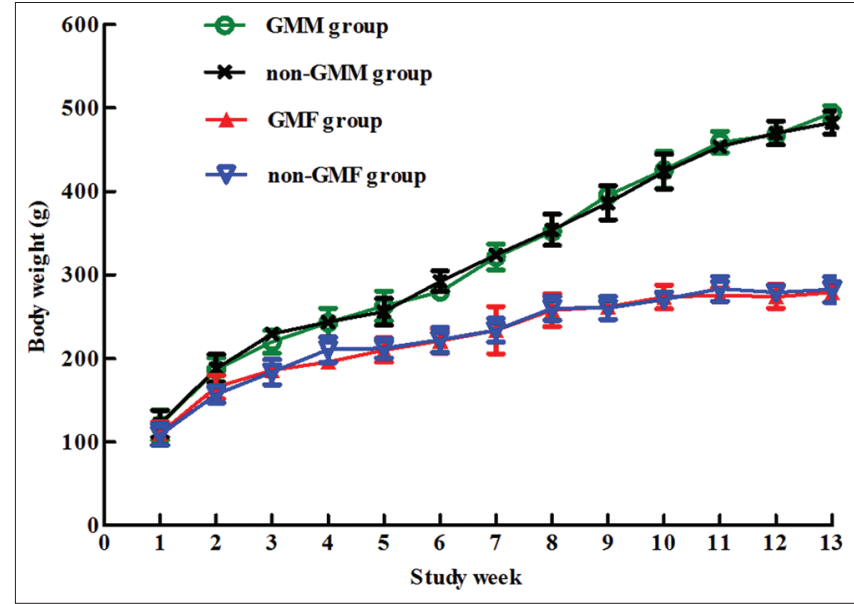

Fig 2. Average weekly body weight of rats. GMF, female rats fed transgenic soybean meal; non-GMF, female rats fed non-transgenic soybean meal; GMM, male rats fed transgenic soybean meal; nonGMM, male rats fed non-transgenic soybean meal.

change or lesion evidence was observed in the tissues or organs of female and male rats exposed to soybean GTS403-2 meal. Therefore, the consumption of soybean GTS403-2 meal was not associated with significant differences in the relative incidence of toxicological histopathological lesions. 


\section{DISCUSSION}

More and more GM crops have been created with the fast development of genetic engineering techniques (Bakhsh and Hussain, 2015; James, 2015). How these novel foods should be evaluated for safety is becoming a crucial issue in the world (Han et al., 2005; Wang et al., 2013; Huang, 2015). In recent years, the GM crop safety has become a popular and hot topic. Although existing scientific evidence suggests that GM crops are similar to their traditional counterparts, the public remains concerned that eating food from GM crops may lead to health problems. Some scientists believe that GM crops have not been fully tested to prove the lack of adverse effects on health of human (Zhang and Shi, 2011; Zhou et al., 2012). Safety assessment derived from GM crops typically requires the substantial equivalence in nutrition to foods obtained from conventional crops. In livestock, the studies of feed utilization, along with comparisons of major nutrients and anti-nutrients, and compositional analyses, are typically conducted to prove substantial equivalence to the majority of regulatory agencies. The 90 -day rodent toxicity study is a typical experimental design to test the food safety from transgenic crops. This type of study provides data on the nutritional performance of crops, but it is a sub-chronic toxicological study.

Currently, the "substantial equivalence" principle has been considered as a food safety evaluation international standard of GM crops (Han et al., 2005; He et al., 2009; Zhou et al., 2011; Wang et al., 2013). Based on this principle, a comparison of nutritional components between the GM crop and their parents is a very important factor in the GM food or feed product safety evaluation (OECD, 1993; He et al., 2009; Zhou et al., 2011; Park et al., 2012; Wang et al., 2013). In our study, the safety of long-term (at least 195-day) dietary exposure to GM GTS40-3-2 herbicide-resistant soybean was assessed by nutritional response and standard toxicological variables in SD rats. The primary nutrients of GM soybean GTS40-3-2 and its non-transgenic parent soybean A5403 were analyzed prior to feeding it to SD rats. Except for crude fat and ash, the data of nutrient composition for the transgenic soybean GTS40-3-2 was within the confines of the literature reported for soybean. Basic nutrient composition demands were fulfilled, these slight differences in crude fat and ash were viewed as minor for soybean (Poulsen et al., 2007; Kilic and Akay, 2008; He et al., 2008). In addition, there was no difference in the nutritional quality of rat diets prepared with 20\% GM GTS40-3-2 soybean meal compared with diets consisting of non-transgenic A5403 soybean meal. All SD rats survived during the test study period, and there were also no treatment-related adverse effects in clinical symptoms, body weight, blood biochemistry, feed consumption, hematology, organ weights, gross events, and histopathology. However, there were minor differences in testis, ovaries, and brain weight between the test and control groups, although they remained within the normal ranges for SD rats. In our study, the nutrient profiles for GM GTS40-3-2 and its parent A5403 showed substantial equivalencies, which were similar to values in published literature. Data obtained in this study indicated that GM GTS40-3-2 is unlikely to cause any adverse clinical or nutritional outcomes in SD rats, compared with its parent cultivar or conventional varieties A5403 for which histories of safe use have been established.

Given that there remain many concerns and debates regarding whether GM crops pose potential risks to humans and animals, it is important to evaluate the safety of transgenic soybean before it is placed on the market, particularly as it will be widely consumed worldwide. If the public perceives that the safety of GM crops has not been adequately evaluated, suspicions will remain. Thus, the question of the effects of some staple foods (such as soybean, maize, and rice) derived from GM crops on the intestinal flora of the consuming animal is of great interest and importance. Therefore, we studied the potential effects of transgenic glyphosate herbicideresistant soybean GTS40-3-2 on SD rats in a long-term and multigenerational feeding study. Based on the results, we believe that it would be beneficial to conduct real-time

Table 5: Hematology (mean value \pm standard deviation, $\mathrm{n}=10$ )

\begin{tabular}{lcccc} 
Hematology & GMM group & Non-GMM group & GMF group & Non-GMF group \\
\hline HGB $(\mathrm{g} / \mathrm{L})$ & $143.35 \pm 7.30$ & $140.9 \pm 7.24$ & $130.30 \pm 11.00$ & $128.62 \pm 8.02$ \\
RBC $\left(10^{12} / \mathrm{L}\right)$ & $8.35 \pm 0.27$ & $8.10 \pm 0.42$ & $9.42 \pm 0.35$ & $9.12 \pm 0.66$ \\
WBC $\left(10^{9} / \mathrm{L}\right)$ & $9.95 \pm 0.67$ & $9.27 \pm 0.50$ & $8.80 \pm 0.92$ & $8.66 \pm 0.70$ \\
LYM $(\%)$ & $60.10 \pm 4.05$ & $59.21 \pm 2.55$ & $52.34 \pm 3.38$ & $52.46 \pm 5.81$ \\
MO $\left(10^{6} / \mathrm{L}\right)$ & $0.20 \pm 0.07$ & $0.22 \pm 0.07$ & $0.20 \pm 0.06$ & $0.21 \pm 0.04$ \\
EO $\left(10^{6} / \mathrm{L}\right)$ & $0.19 \pm 0.02$ & $0.20 \pm 0.05$ & $0.18 \pm 0.05$ & $0.18 \pm 0.03$ \\
PLT $\left(10^{9} / \mathrm{L}\right)$ & $288.52 \pm 21.10$ & $291.98 \pm 14.22$ & $228.69 \pm 35.90$ & $217.12 \pm 13.00$
\end{tabular}

HGB, hemoglobin; RBC, red blood cells; LYM, lymphocytes; WBC, white blood cells; EO, eosinophils; MO, monocytes; PLT, platelet count; GMF, female rats fed transgenic soybean meal; non-GMF, female rats fed non-transgenic soybean meal; GMM, male rats fed transgenic soybean meal; non-GMM, male rats fed non-transgenic soybean meal 
polymerase chain reaction assessments of transgenic crops, in order to strengthen the safety evaluation process, before placing them on the market.

Overall, no treatment-related adverse effects were found in SD rats consuming transgenic GTS40-3-2 soybean meal. Therefore, the results in this study fully show that GM GTS40-4-2 soybeans are safe to consume.

\section{CONCLUSION}

Under the given experimental conditions, there were no adverse effects or significant differences in SD rats after consuming a diet formulated with the transgenic glyphosate herbicide-resistant soybean GTS40-3-2 meal, compared with a diet formulated with the parent A5403 soybean meal.

\section{ACKNOWLEDGEMENTS}

This work was supported by the China National Natural Science Foundation (grant nos. 31372497), Shanxi Province Science and Technology Key Program (grant no. 20140311025-3), and the Science and Technology Innovation Fund of Shanxi Agricultural University (grant nos. 2016ZZ09).

\section{Author contribution}

Study concepts and design: J. Y.; material preparation: J. Y; J. Z; Z. S.; literature research: J. Y; Z. T.; data analysis/ interpretation, manuscript preparation and definition of intellectual content: J. Y; J. Z.; manuscript editing: J. Y; J. W.; manuscript revision/review: J. W.; and manuscript final version approval: J. Y; Z. T; J. Z.; Z. S.; J. W.; All authors read and approved the manuscript.

\section{REFERENCES}

Appenzeller, L. M., S. M. Munley, D. Hoban, G. P. Sykes, L. A. Malley and B. Delaney. 2008. Subchronic feeding study of herbicidetolerant soybean DP-356043-5 in sprague-dawley rats. Food Chem. Toxicol. 46: 2201-2213.

Bakhsh, A. and T. Hussain. 2015. Engineering crop plants against abiotic stress: Current achievements and prospects. Emir. J. Food Agric. 27(1): 24-39.

Chinese Standard GB14924.3-2010. Experimental Animal (mice and rat) Feeds. Standards Press of China, Beijing, China.

Chinese Standard GB15193.13-2003. The Chinese Toxicology Assessment Procedures and Methods for Food Safety. Standards Press of China, Beijing, China.

Chinese Standard GB/T5009.6-2003. Method for Determination of Fat in Foods. Standards Press of China, Beijing, China.

Chinese Standard GB GB/T5009.8-2008. Method for Determination of Dietary Fiber in Foods. Standards Press of China, Beijing, China.

Chinese Standard GB/T5009.3-2010. Method for Determination of
Moisture in Foods. Standards Press of China, Beijing, China.

Chinese Standard GB/T5009.4-2010. Method for Determination of Ash in Foods. Standards Press of China, Beijing, China.

Chinese Standard GB/T5009.5-2010. Method for Determination of Protein in Foods. Standards Press of China, Beijing, China.

Chukwudebe, A., L. Privalle, A. Reed, C. Wandelt, D. Contri, M. Dammann, S. Groeters, U. Kaspers, V. Strauss and B. van Ravenzwaay. 2012. Health and nutritional status of wistar rats following subchronic exposure to CV127 soybeans. Food Chem. Toxicol. 50: 956-971.

Dryzga, M.D., B. L. Yano, A. K. Andrus and J. L. Mattsson. 2007. Evaluation of the safety and nutritional equivalence of a genetically modified cottonseed meal in a 90-day dietary toxicity study in rats. Food Chem. Toxicol. 45: 1994-2004.

Duke, S. O. 2005. Taking stock of herbicide-resistant crops ten years after introduction. Pest Manag. Sci. 61: 211-218.

FAO. 1996. Biotechnology and Food Safety. Report of a Joint FAO/ WHO Consultation. FAO Food and Nutrition Paper No. 61. Food and Agriculture Organization of the United Nations, Rome, Italy.

FAO/WHO. 2000. Safety Aspects of Genetically Modified Foods of Plant Origin. Report of a Joint FAO/WHO Expert Consultation on Foods Derived from Biotechnology, Geneva, Switzerland, 29 May-2 June 2000. Food and Agriculture Organization of the United Nations, Rome, Italy

Han, J. H., Y. X. Yang, S. R. Chen, Z. Wang, X. L. Yang, G. D. Wang and J. H. Men. 2005. Comparison of nutrient composition of parental rice and rice genetically modified with cowpea trypsin inhibitor. China J. Food Comp. Anal. 18(4): 297-302.

He, X. Y., K. L. Huang, X. Li, W. Qin, B. Delaney and Y. B. Luo. 2008. Comparison of grain from corn rootworm resistant transgenic DAS-59122-7 maize with no transgenic maize grain in a 90-day feeding study in sprague-dawley rats. Food Chem. Toxicol. 46: 1994-2002.

He, X. Y., M. Z. Tang, Y. B. Luo, X. Li and S. S. Cao. 2009. A 90-day toxicology study of transgenic lysine-rich maize grain (Y642) in sprague-dawley rats. Food Chem. Toxicol. 47: 425-432.

Huang, D. F. 2015. Review of transgenic crop breeding in China. Chin. J. Biotech. 31(6): 892-900.

James, C. 2013. Global Status of Commercialized Biotech/GM Crops: 2013. ISAAA Brief No. 46. ISAAA, Ithaca, NY.

James, C. 2015. Global status of commercialized Biotech/GM Crops: 2014. China Biotech. 35(1): 1-14.

Kilic, A. and M. T. Akay. 2008. A three-generation study with genetically modified bt corn in rats: Biochemical and histopathological investigation. Food Chem. Toxicol. 6: 1164-1170.

Kroghsbo, S., C. Madsen, M. Poulsen, M. Schrøder, P. H. Kvist, M. Taylor, A. Gatehouse, Q. Shu and I. Knudsen. 2008. Immunotoxicological studies of genetically modified rice expressing PHA-E lectin or bt toxin in wistar rats. Toxicology. 245: 24-34.

Lu, C. B., J. Cai, Q. L. Qi and Y. Y. Wei. 2014. Effects of glyphosateresistant transgenic soybean on male mice testis. J. Jinan Univ. (Natural Science \& Medicine Edition) 35(3): 245-259.

Noteborn, H. P. J., M. E. Bienenmann-Ploum, J. H. J. van den Berg, G. M. Alink, L. Zolla, A. Reynaerts, M. Pensa and H. A. Kuiper. 1995. Safety assessment of the Bacillus thuringiensis insecticidal crystal protein CRYIA (b) expressed in transgenic tomatoes. J. Am. Chem. Soc. 12: 134-147.

OECD. 1993. Safety Evaluation of Foods Produced by Modern Biotechnology, Concepts and Principles. Organization for Economic Cooperation and Development, Paris, France. 
OECD. 1996. Food Safety Evaluation. Organization for Economic Cooperation and Development (OECD), Paris, France.

OECD. 1997. Report of the OECD Workshop on the Toxicological and Nutritional Testing of Novel Foods. Aussois, France, 5-8 March 1997. Organization for Economic Cooperation and Development (OECD), Paris, France.

Park, S. Y., S. M. Lee, J. H. Lee, H. S. Ko, S. J. Kweon, S. C. Suh, K. S. Shin and J. K. Kim. 2012. Compositional comparative analysis between insect-resistant rice (Oryza sativa L.) with a synthetic Cry1Ac gene and its non-transgenic counterpart. Plant Biotechnol. Rep. 6: 29-37.

Poulsen, M., S. Kroghsbo, M. Schrøder, A. Wilcks, H. Jacobsen, A. Miller, T. Frenzel, J. Danier, M. Rychlik, Q. Shu, K. Emami, D. Sudhakar, A. Gatehouse, K. H. Engel and I. Knudsen. 2007. A 90-day safety study in wistar rats fed genetically modified rice expressing snowdrop lectin Galanthus nivalis (GNA). Food Chem. Toxicol. 245(3): 350-363.

Sissener, N. H., A. M. Bakke, J. Gu, M. H. Penn, E. Eie, A. Krogdahl, M. Sanden and G. I. Hemre. 2009. An assessment of organ and intestinal histomorphology and cellular stress response in Atlantic salmon (Salmo salar L.) fed genetically modified roundup ready $\circledast$ soy. Aquaculture. 298: 101-110.

Wang, E. H., Z. Yu, J. Hu and H. B. Xu. 2013. Effects of 90-day feeding of transgenic bt rice TT51 on the reproductive system in male rats. Food Chem. Toxicol. 62: 390-396.

WHO. 1991. Strategies for Assessing the Safety of Foods Produced by Biotechnology. Report of a Joint FAO/WHO Consultation. World Health Organization, Geneva, Switzerland.

WHO. 1995. Application of the Principles of Substantial Equivalence to the Safety Evaluation of Foods and Food Components from Plants Derived from Modern Biotechnology. Report of a WHO Workshop. World Health Organization, Geneva, Switzerland.

Zhang, W. and F. Shi. 2011. Do genetically modified crops affect animal reproduction? A review of the ongoing debate. Animal. 5(7): 1048-1059.

Zhou, X. H., Y. Dong, X. Xiao, Y. Wang, Y. Xu, B. Xu, W. D. Shi, Y. Zhang, L. J. Zhu and Q. Q. Liu. 2011. A 90-day toxicology study of high-amylose transgenic rice grain in sprague-dawley rats. Food Chem. Toxicol. 49: 3112-3118.

Zhou, X. H., Y. Dong, Y. Wang, X. Xiao, Y. Xu, B. Xu, X. Li, X. S. Wei and $Q$. Q. Liu. 2012. A three generation study with high-lysine transgenic rice in sprague-dawley rats. Food Chem. Toxicol. 50: 1902-1910.

Zhu, Y. X., X. Y. He, Y. B. Luo, S. Y. Zou, X. Zhou, K. L. Huang and W. T. Xu. 2013. A 90-day feeding study of glyphosate-tolerant maize with the G2-aroA gene in sprague-dawley rats. Food Chem. Toxicol. 51: 280-287. 